\title{
Formulation Design and In vitro Evaluation of Berberine- Loaded Self-Nanoemulsifying Drug Delivery System
}

\author{
Zhongcheng Ke*, Zhi-Ping Zhu, Zhi-Yuan Xu, Chao Fang and Shang-Qing Hu \\ College of Chemistry and Chemical Engineering, Huangshan University, Huangshan, 245041, China
}

*For correspondence: Email: xiaoke1020@126.com; Tel: +865592546612

Received: 19 December 2014

Revised accepted: 16 April 2015

\begin{abstract}
Purpose: To improve the oral bioavailability of berberine using a new self-nanoemulsifying drug delivery system (SNEDDS).

Methods: Berberine SNEDDS was designed using solubility studies and phase diagram construction. A series of tests were carried out to study the effect of oil content, dilution, and drug loading on particle size. The morphology of the nanoemulsion was examined with a transmission electron microscope. Subsequently, the optimized berberine SNEDDS was investigated for in vitro release and compared with two marketed products (Xiaowei capsules and Beike tablets)

Results: The optimized formulation, composed of berberine of $6.0 \%$, castor oil of $18.8 \%$, Tween 20 of $37.6 \%$, and glycerol of $37.6 \%$, gave a mean particle size of approximately $60 \mathrm{~nm}$, and was not affected by dilution. The optimized formulation released $90 \%$ berberine in $20 \mathrm{~min}$, while the reference products, Xiaowei capsules and Beike tablets, required $2 \mathrm{~h}$ to release $90 \%$ of berberine.

Conclusion: The optimized formulation of SNEDDS is a potential alternative oral dosage form for improving oral absorption of berberine.
\end{abstract}

Keywords: Berberine, Pseudoternary phase diagram, Self-nanoemulsifying, SNEDDS, Drug release

Tropical Journal of Pharmaceutical Research is indexed by Science Citation Index (SciSearch), Scopus, International Pharmaceutical Abstract, Chemical Abstracts, Embase, Index Copernicus, EBSCO, African Index Medicus, JournalSeek, Journal Citation Reports/Science Edition, Directory of Open Access Journals (DOAJ), African Journal Online, Bioline International, Open-J-Gate and Pharmacy Abstracts

\section{INTRODUCTION}

Self-nanoemulsifying drug delivery systems (SNEDDS) are isotropic and thermodynamically stable solutions consisting of oil, surfactant, cosurfactant and drug that can self-emulsify quickly in the stomach aqueous contents. Thus, it introduces the drug in solution within nanosized oil droplets [1-3]. Being nanosized, SNEDDS not only improves drug solubilization but also enhances release and absorption properties, due to the resulting small droplet size thus providing a large interfacial surface area [4-6].

Berberine is one of the major components of Coptis chinensis Franch. It has been clinically applied for decades because of its significant effect. Berberine, a lipophilic antibacterial agent [7], also presents antidiarrhea [8], hypoglycemic [9] and antitumor [10] properties. The current commercially marketed dosage forms are tablets and capsules. Both of these exhibit low and erratic oral bioavailability, which is mainly due to the poor dissolution of the drug.

The purpose of the present study was to formulate an oral delivery system for berberine using SNEDDS. The release of berberine from the nanoemulsion formulation was compared to that of commercially available tablets and capsules. 


\section{EXPERIMENTAL}

\section{Materials}

Berberine was purchased from Xingren pharmaceutical Co., Ltd (Zhengzhou, China). Oleic acid, isopropyl myristate, castor oil and cod liver oil were purchased from east sea pharmaceutical Co., Ltd (Qingdao, China). Tween-20, tween-80, OP emulsifier, isopropyl alcohol, glycerol, ethanol were purchased from Sinopharm Chemical Reagent Co., Ltd (Beijing, China). All the other chemicals used were of analytical reagent grade. Double-distilled water was used throughout the whole study.

\section{Analysis of berberine}

The concentration of berberine was determined by Agilent 1200 high performance liquid chromatograph with a UV detector. The chromatographic column was Alltima C18 (250 × $4.6 \mathrm{~mm}$ ) with $5 \mu \mathrm{m}$ particle size. The mobile phase consisted of acetonitrile/0.05 M $\mathrm{KH}_{2} \mathrm{PO}_{4} /$ triethylamine (50:50:0.5, v/v/v) at a flow rate of $1.0 \mathrm{~mL} / \mathrm{min}$. A volume of $20 \mu \mathrm{L}$ of each solution was injected into the HPLC and the eluent was monitored at $345 \mathrm{~nm}$. The retention time of berberine was $8.3 \pm 0.05 \mathrm{~min}$ at ambient room temperature. The mean calibration curve was given by the equation, $y=36.1 x-0.65, r=$ 0.9998 , where $y$ represents peak area and $x$ represents the concentration (ug/mL) of berberine. The method was validated for accuracy precision, specificity and solution stability.

\section{Solubility studies}

The solubility of berberine in various solvents was determined as follows: An excess amount of berberine was added into $5 \mathrm{~mL}$ of each vehicles and mixture were kept in sealed vials. After capping the tubes, the mixtures were shaken at $40{ }^{\circ} \mathrm{C}$ for $24 \mathrm{~h}$ to achieve dissolution equilibrium. Then, after standing for $24 \mathrm{~h}$ and reaching equilibrium at $25^{\circ} \mathrm{C}$, each vial was centrifuged at $4,000 \mathrm{rpm}$ for $10 \mathrm{~min}$ using a centrifuge (Eppendorf Centrifuge 5810). Undissolved berberine was removed by a membrane filter $(0.45 \mu \mathrm{m})$. The samples were diluted with methanol and passed through a $0.22 \mu \mathrm{m}$ cellulose nitrate membrane. The concentration of dissolved berberine was determined by HPLC.

\section{Construction of phase diagram}

On the basis of solubility study, castor oil was selected as a lipid phase, Tween20 and glycerol were used as a surfactant and cosurfactant, respectively. To determine the concentration of components for the existing range of SNEDDS, pseudoternary phase diagram was constructed using water titration method at ambient temperature $\left(25^{\circ} \mathrm{C}\right)$. Surfactant and cosurfactant were mixed in different volume ratios (1:3, 1:2, $1: 1,2: 1$ and $3: 1)$. Oil and surfactant/co-surfactant mixture $(\mathrm{O}-\mathrm{S} / \mathrm{Co})$ were mixed thoroughly in different volume ratios $(1: 9,1: 8,1: 7,1: 6,1: 5,1: 4$, $1: 3,1: 2,1: 1,2: 1,3: 1,4: 1,5: 1,6: 1,7: 1,8: 1$ and $9: 1)$. The mixtures of oil, surfactant and cosurfactant at certain weight ratios were titrated with deionized water by drop wise addition under gentle addition. Resulting mixture was observed for their transparency until titration endpoint (turbid was observed). At this point, amount of water, oil, surfactant and cosurfactant added were noted, and the values were used to construct pseudo-ternary plot. Monophasic, clear, low viscous and non-birefringent systems were considered as nanoemulsion (NE) and shown as NE region.

\section{Effect of oily phase content on mean droplet size}

Based on the results of phase diagram, $\mathrm{Km}$ is set as $1: 1$ in Table 1 , a series of blank SNEDDS were prepared with varying oil content to learn the effect of oil content on mean globule size. Briefly, oil, surfactant and cosurfactant were mixed by stirring to form homogenous mixture. Then, $10 \mathrm{mg}$ mixture was dispersed in $500 \mathrm{~mL}$ double distilled water, droplet size was determined immediately after dilution.

\section{Preparation of berberine SNEDDS}

Berberine was dissolved into oil in glass vials. Surfactant and cosurfactant were accurately weighed and were added in.

Table 1: Composition of SNEDDS formulations with varying oil content

\begin{tabular}{lcccccc}
\hline \multirow{2}{*}{ Ingredients } & \multicolumn{7}{c}{ Formulation $(\mathbf{g})$} \\
\cline { 2 - 7 } & F1 & F2 & F3 & F4 & F5 & F6 \\
\hline Tween20+Glycerol (at ratio of 1:1) & 44 & 42 & 40 & 38 & 36 & 34 \\
Castor oil & 6 & 8 & 10 & 12 & 14 & 16 \\
\hline
\end{tabular}


Components were mixed and heated $\left(40-50{ }^{\circ} \mathrm{C}\right)$ to form SNEDDS, a homogenous mixture. Berberine loaded SNEDDS were stored at room temperature for further characterization.

\section{Effect of berberine loading}

Effect of berberine loading on physical stability of nanoemulsions was studied using optimized composition i.e. formulation F3. Accordingly, a series of SNEDDS was prepared with varying amount of berberine $(1.0,2.0,4.0,6.0,8.0,10.0$ $\%$ ). SNEDDS, $10 \mathrm{mg}$ was diluted with $500 \mathrm{~mL}$ of different media. Mean droplet size of nanoemulsions was determined.

\section{Determination of droplets size distribution}

The droplet size, size distribution were analysed by dynamic light scattering with particle size apparatus (Malvern Zetasizer 3000 HS). Berberine SNEDDS were diluted with deionized water (1:50) in a drop-wise manner at $25{ }^{\circ} \mathrm{C}$ under gentle shaking. After equilibrium droplet size and zeta potential were recorded.

\section{Dilution studies}

Dilution study was done to access the effect of dilution on SNEDDS. In this study, Optimized formulation was subjected to various dilutions(1:20,1:50,1:100) with deionized water, then rapid form nanoemulsion, which is clear or slightly bluish in appearance or not.

\section{Thermodynamic stability studies of berberine SNEDDS}

The objective of the phase separation research is to evaluate thermodynamic stability of optimized berberine SNEDDS. SNEDDS were diluted with aqueous medium (1:50) and centrifuged at 4,000 rpm for $30 \mathrm{~min}$ and formulation were observed visually for phase separation and drug precipitation.

\section{Transmission electron microscopy}

The morphology of SNEDDS was observed using a transmission electron microscope (TEM; JEM-1200EX, Japan). SNEDDS was diluted with deionized water at 1:50 and mixed by slight shaking. A drop of the diluted samples was directly deposited on a holey film grid and then stained with one drop of $2 \%$ aqueous solution of phosphotungstic acid (PTA), and allowed to dry before observation under the electron microscope.

\section{In vitro dissolution profile}

Berberine SNEDDS was evaluated for in vitro release by using USP XXIII apparatus I at $37 \pm$ $0.5^{\circ} \mathrm{C}$. The revolution speed of the paddle was kept constant at $100 \mathrm{rpm}$, and $500 \mathrm{~mL} 0.1 \mathrm{M}$ hydrochloride solution was chosen as the dissolution media. During study, $5 \mathrm{~mL}$ aliquots removed at $0,5,10,20,30,60,90$ and $120 \mathrm{~min}$, and filtered through $0.45 \mu \mathrm{m}$ membrane filters. Amount of drug released was determined using HPLC method. The removed volume was replaced each time with $5 \mathrm{~mL}$ of fresh medium.

\section{Statistical analysis}

SPSS $18 \circledast$ software was used to analyze the data. All results were expressed as the mean \pm standard deviation. Dissolution data of the different formulations were compared by oneway analysis of variance (ANOVA). $P<0.05$ was considered to be statistically significant.

\section{RESULTS}

Solubility of berberine in various excipients are shown in Table 2. Among oil phase, castor oil solubilize maximum amount of berberine, whereas in surfactants or cosurfactants, Tween20 and glycerol exhibited good solubility for berberine.

Table 2: Solubility results of berberine in various excipients (mean $\pm S D ; n=3$ )

\begin{tabular}{lccc}
\hline Oil phase & Solubility $(\mathbf{m g} / \mathbf{m L})$ & Surfactant and cosurfactant & Solubility $(\mathbf{m g} / \mathbf{m L})$ \\
\hline Isopropyl myristate & $0.11 \pm 0.02$ & Cremophor EL & $1.19 \pm 0.16$ \\
Oleic acid & $0.26 \pm 0.03$ & Tween-80 & $0.36 \pm 0.14$ \\
Castor oil & $0.38 \pm 0.04$ & Tween-20 & $1.24 \pm 0.13$ \\
Cod liver oil & $0.16 \pm 0.03$ & Glycerol & $2.87 \pm 0.22$ \\
& & Ethanol & $2.84 \pm 0.19$ \\
& & Isopropyl alcohol & $0.43 \pm 0.05$ \\
\hline
\end{tabular}


The pseudo-ternary phase diagrams of the formulation composed of castor oil, Tween 20, glycerol and water are described.Pseudo-ternary plot was constructed using Oringe 6.0 software. Figures show ternary phase diagrams for castor oil - Tween20 - glycerol systems, respectively. Nanoemulsion area was highest at $\mathrm{Km}=1$. Hence, surfactant to cosurfactant ratio was maintained at 1:1. The size of nanoemulsion region was compared, larger the size, greater is the self-nanoemulsification efficiency.

Effects of oil concentration on mean droplet size are shown in Fig 1. SNEDDS formulations F1 and F2 were found rapid to dilution and did not show any phase separation after $24 \mathrm{~h}$. From phase diagram, it was evident that castor oil Tween20 - glycerol system can incorporate more than $20 \% \quad w / w$ of oil phase. However, compositions with such high oil content may not be necessarily stable. Hence, SNEDDS with varying oil content at $\mathrm{Km}=1$ were formulated to identify optimum oil concentration that can yield nanoemulsion of desired droplet size. It is evident that up to $20 \% \mathrm{w} / \mathrm{w}$ concentration of castor oil (formulations F1, F2, F3), mean globule size was $<50 \mathrm{~nm}$. However, increase in oil content above $24 \%$ and mean droplet size increased considerably. Formulations F5, F6 that contained oil phase $28 \%$ and above exhibited globule size greater than $100 \mathrm{~nm}$. Based on this study, formulation $\mathrm{F} 3$ that contained $20 \% \mathrm{w} / \mathrm{w}$ oil was selected for further study.

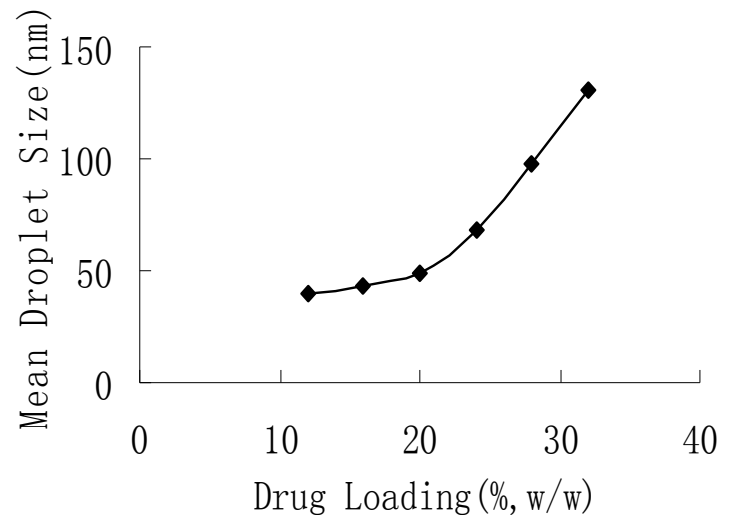

Fig 1: Effect of oil content on mean droplet size $(n=3)$

Effect of berberine loading on mean droplet size is shown in Fig 2. Droplet size experiments showed that incorporation of berberine in SNEDDS does not have any impact on droplet size when its concentration was up to $6.0 \% \mathrm{w} / \mathrm{w}$. However, mean globule size increased significantly when concentration of berberine was $8.0 \% \mathrm{w} / \mathrm{w}$ and above.

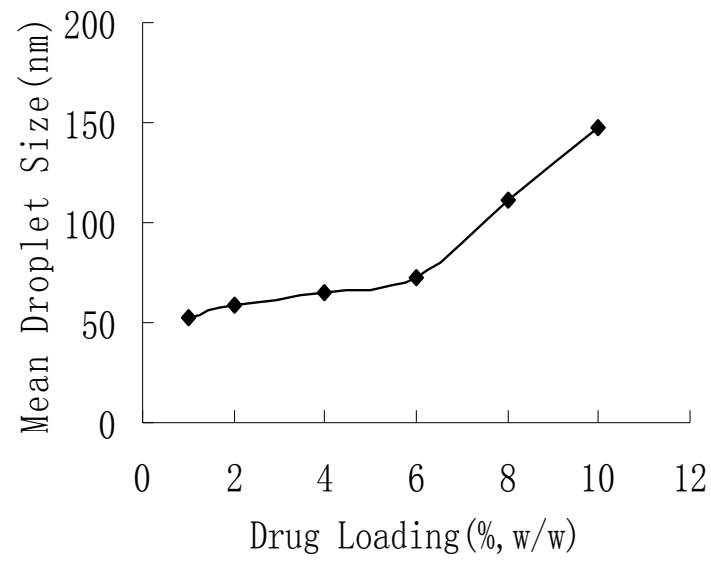

Fig 2: Effect of berberine loading on mean droplet size $(n=3)$

Accordingly, berberine SNEDDS formulation was selected for further experiments and its compositions were as follows: berberine $12.8 \mathrm{~g}$, castor oil $40 \mathrm{~g}$, Tween $2080 \mathrm{~g}$ and glycerol $80 \mathrm{~g}$.

Optimized berberine SNEDDS were diluted with deionized water in a drop-wise manner at $25^{\circ} \mathrm{C}$ under gentle shaking. After equilibrium droplet size was $58.4 \mathrm{~nm}$, polydispersity index was 0.26 , and zeta potential was $-12.9 \pm 0.5 \mathrm{mv}$.

The influence of increasing the dilution factor from $(1: 10,1: 50$ and 1:100) was evaluated, they all rapidly formed clear nanoemulsion, In all cases, increased dilution resulted in the nanoemulsion remaining with the same clarity. Phase separation and drug precipitation were not observed in sample solution during the stability studies.

The morphology of the nanoemulsion was examined with a transmission electron microscope. The droplet on the nanoemulsion appears dark with the bright surroundings. TEM photographs (Fig 3 ) further conformed that the globules are approximate spherical in shape.

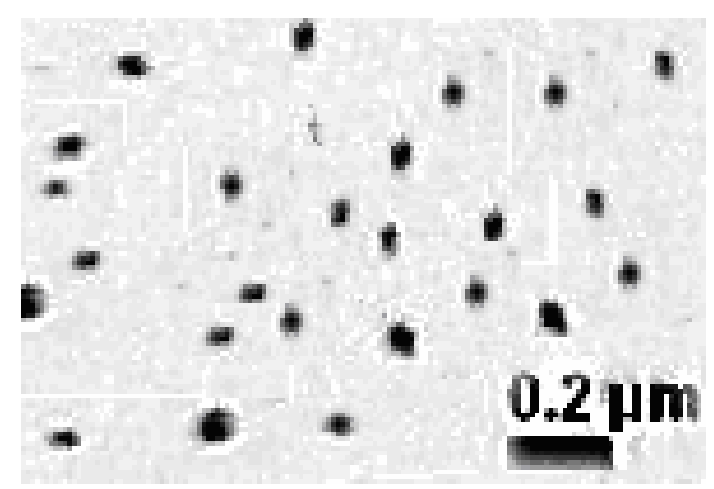

Fig 3: TEM photograph 
In vitro dissolution profile of optimized berberine SNEDDS in comparison to its commercial capsules (Xiaowei, China) and market tablets (Beike, China) in $0.1 \mathrm{M}$ hydrochloride solution is shown in Fig 4. SNEDDS release $90 \%$ berberine in 20 min in dissolution media, Xiaowei capsules and Beike tablets required $2 \mathrm{~h}$ to release $90 \%$ of berberine $(p<0.05)$. Dramatic increase in the rate of release of berberine from SNEDDS compared to commercial capsules and tablets can be attributed to its quick dispersability and ability to keep drug in solubilized state.

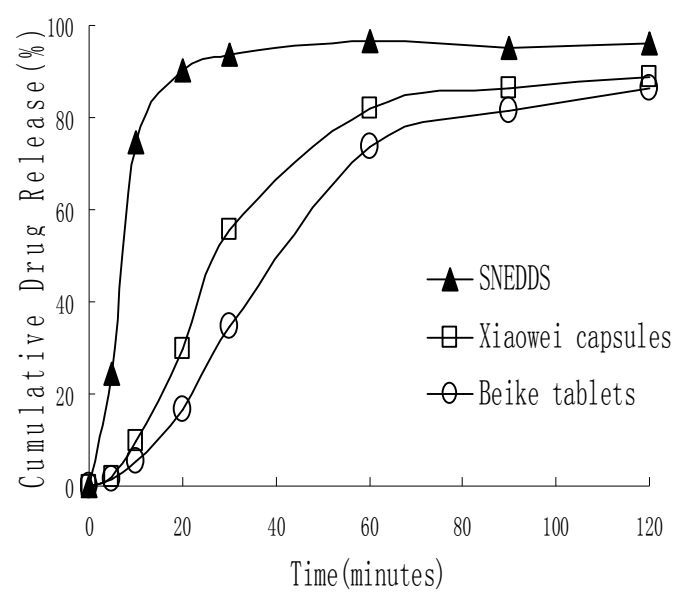

Fig 4: Release profiles of berberine from various samples (mean $\pm S D, n=3$ )

\section{DISCUSSION}

As it is important to achieve optimum drug loading, solubility study was aimed to identify suitable SNEDDS components that possess good solubilizing capacity. So the solubility of berberine in various solvents was determined.

Self-nanoemulsifying drug-delivery systems offer the potential for enhancing the absorption and hence the oral bioavailability of lipophilic drugs. The primary mechanisms are presenting the drug greater surface area or in solubilized form in vivo, and increasing mucosal permeability, increasing incorporation into lipoproteins, then secreted into the lymphatics, which circumvents the liver, thus reducing the hepatic first-pass metabolism [11].

Enhancement of bioavailability of berberine SNEDDS compared with Beike tablets and Xiaowei capsules may be due to effects of the large quantities of surfactant and cosurfactant used which include improved mucosal permeability, smaller lipid droplets and greater surface area.
In view of the toxicity of Tween 20, it was substituted with Cremophor EL. Thus, the optimized formulation was as follows: berberine $(6.0 \% \mathrm{w} / \mathrm{w})$, castor oil $(18.8 \% \mathrm{w} / \mathrm{w})$, Cremophor EL $(37.6 \% \mathrm{w} / \mathrm{w})$ and glycerol $(37.6 \% \mathrm{w} / \mathrm{w})$. When diluted with water (1:100), this modified SNEDDS spontaneously formed small particles with average droplet size of about $62 \mathrm{~nm}$, Dissolution experiments showed that the modified SNEDDS released $90 \%$ berberine in 20 $\min$.

\section{CONCLUSION}

A self-nanoemulsifying drug delivery system for berberine has been successfully achieved in this study. This delivery system can potentially be employed to maximize the antibacterial capacity of a drug. The technique used can potentially be used also to prepare other solid selfnanoemulsifying drug delivery systems.

\section{ACKNOWLEDGEMENT}

The authors gratefully acknowledge the financial support for this study by Anhui Universities Provincial Natural Science Key Research Project, Huangshan, China (no. KJ2014A242) and Provincial Training Programs of Innovation and Entrepreneurship for Undergraduates, Huangshan, China (no. AH201310375052).

\section{REFERENCES}

1. Akhter MH, Ahmad A, Ali J, Mohan G. Formulation and Development of CoQ10-Loaded s-SNEDDS for Enhancement of Oral Bioavailability. J Pharm Innov, 2014; 9:121-131.

2. Elgart A, Cherniakov I, Aldouby Y. Improved Oral Bioavailability of BCS Class 2 Compounds by Self Nano-Emulsifying Drug Delivery Systems (SNEDDS): The Underlying Mechanisms for Amiodarone and Talinolol. Pharm Res, 2013; 30: 3029-3044.

3. Guo F, Zhong HJ, He J, Xie BG, Liu F, Xu HL, Liu MM, Xu CL. Self-microemulsifying Drug Delivery System for Improved Oral Bioavailability of Dipyridamole: Preparation and Evaluation. Arch Pharm Res, 2011; 34(7):11137-1122.

4. Yoo JH, Shanmugam S, Thapa $P$, Lee ES, Balakrishnan $P$, Baskaran R, Yoon SK, Choi HG, Yong CS, Yoo BK, Han K. Novel Self-nanoemulsifying Drug Delivery System for Enhanced Solubility and Dissolution of Lutein. Arch Pharm Res, 2010; 33(3): 417-426.

5. Shahba AAW, Mohsin K, Alanazi1 FK. Novel SelfNanoemulsifying Drug Delivery Systems (SNEDDS) for Oral Delivery of Cinnarizine: Design, Optimization, and In-Vitro Assessment. AAPS, 2012; 13(3): 967977.

Trop J Pharm Res, May 2015; 14(5): 751 
6. Jain AK, Thanki K, Jain S. Solidified SelfNanoemulsifying Formulation for Oral Delivery of Combinatorial Therapeutic Regimen: Part I. Formulation Development, Statistical Optimization, and In Vitro Characterization. Pharm Res, 2014; 31: 923-945.

7. Zhang $Y, L i X$, Zhang $Q, L i J$, Ju J, Du N, Liu X, Chen X, Cheng $F$, Yang L, Xu C, Wei Y, Lu Y, Yang $B$. Berberine hydrochloride prevents postsurgery intestinal adhesion and inflammation in rats. $J$ Pharmacol Exp Ther, 2014; 349(3): 417-426.

8. Xia XC, Wang H, Niu X, Wang HJ, Liu ZQ, Liu YM, Qi ZM, Wang SY, Liu SX, Liu SS. Assessment of the antidiarrhea function of compound Chinese herbal medicine Cangpo Oral Liquid. Afr J Tradit Complement Altern Med, 2013; 11(1): 140-147.
9. Perez-Rubio KG, Gonzalez-Ortiz M, Martinez-Abundis E, Robles-Cervantes JA, Espinel-Bermudez MC. Effect of berberine administration on metabolic syndrome, insulin sensitivity, and insulin secretion. Metab Syndr Relat Disord, 2013; 11(5): 366-369.

10. $\mathrm{Li} C H$, Wu DF, Ding $H$, Zhao Y, Zhou $K Y, X U D F$. Berberine hydrochloride impact on physiological processes and modulation of twist levels in nasopharyngeal carcinoma CNE-1 cells. Asian Pac J Cancer Prev, 2014; 15(4): 1851-1857.

11. Porter CJ, Trevaskis NL, Charman WN. Lipids and lipidbased formulations: Optimizing the oral delivery of lipophilic drugs. Nat Rev Drug Discov, 2007; 6: 231248. 